\title{
Vorhersage des Umschulungserfolgs durch die Berufseignungsdiagnostik
}

\section{Predicting the Success of Occupational Retraining Using the Occupational Aptitude Test Battery}

\author{
Autor \\ L. Kreuzpointner \\ Institut \\ Institut für Psychologie, Lehrstuhl für Psychologie VI - Prof. Lukesch, Universität Regensburg
}

Schlüsselwörter
Abklärung der
beruflichen Eignung und
Arbeitserprobung
- Prognose von
Umschulungserfolg und
Ausbildungserfolg
Regressionsmodell
Key words
assessment of occupational
aptitude
prediction of success of
occupational retraining in
office management
regression model

Schlüsselwörter

beruflichen Eignung und

Arbeitserprobung

Prognose von

Umschulungserfolg und

Ausbildungserfolg

Key words

- assessment of occupational aptitude

occupational retraining in

- regression model

\section{Zusammenfassung \\ $\nabla$}

In der vorliegenden Arbeit wird der multiple Zusammenhang zwischen den Ergebnissen der Leistungstests, die im Rahmen der Abklärung der beruflichen Eignung und Arbeitserprobung durch das Berufsförderungswerk Eckert erhoben wurden, und dem Erfolg der Umschulung im kaufmännischen Bereich untersucht. Es zeigt sich, dass wenige Variablen nötig sind, um ein Maximum an Varianz der Prognosekriterien Umschulungsnoten und Abschlussnoten der IHKPrüfung zu klären. Durch vier unterschiedlich zusammengestellte Regressionsmodelle können je rund 25\% der Varianz des Umschulungserfolgs erklärt werden. Bedeutsame Variablen sind hierbei Indikatoren für sprachliche Fähigkeiten und für mathematisches Basiswissen. Ein Maß für nonverbale Intelligenz muss in jedem Modell als Mediatorvariable mit negativer Wirkrichtung berücksichtigt werden. Es zeigt sich, dass zur Vorhersage des Umschulungserfolgs einer kaufmännischen Ausbildung vor allem der Erhalt des schulischen Wissens (v.a. Rechtschreibung und grundlegende Mathematikkenntnisse) und weniger das Maß der allgemeinen Intelligenz beachtet werden muss.

\section{Abstract \\ $\nabla$}

In vocational retraining centres, a test battery including several performance tests is generally administered to assess the occupational aptitude of rehabilitants and to predict their success in occupational retraining. This paper presents the multiple regressions of a set of achievement scores on "grades of retraining" and "grades of final examination", respectively, concerning retraining to become an office management assistant. It was shown that only few variables are adequate to clarify a maximum of variance of the criterions. Four different regression models were identified; each of them could clarify about $25 \%$ of variance. Significant predictors were indicators for verbal skills and basic numeracy. In each model a measurement for nonverbal intelligence had to be taken into account as a suppressor variable. To put all in a nutshell, in order to predict the success of vocational retraining to become an office management assistant it is more important to focus on strengthening school knowledge than on general intelligence.
Bibliografie

DOI 10.1055/s-0029-1202293

Rehabilitation 2009;

48: $1-8$

(c) Georg Thieme Verlag KG

Stuttgart · New York

ISSN 0034-3536

Korrespondenzadresse

\section{Kreuzpointner}

Universität Regensburg

Institut für Psychologie

93040 Regensburg

ludwig.kreuzpointner@

psychologie.uni-regensburg.de

\section{Einleitung}

\section{$\nabla$}

Im Rahmen der Abklärung der beruflichen Eignung und Arbeitserprobung sowohl durch die Berufsförderungswerke als auch durch die Bundesagentur für Arbeit wird in der Regel eine Testbatterie aus Intelligenz-, Konzentrations- und Gedächtnistests, Tests für spezielle Fähigkeiten und Kenntnisse sowie Persönlichkeits- und Interessentests verwendet. Diese Testung dient zum einen der Bestimmung von für den Probanden geeigneten Umschulungs- und Ausbildungsrichtungen. Zum anderen soll damit auch vorherge- sagt werden, ob und inwieweit der Kandidat den schulischen Herausforderungen einer Umschulung gewachsen sein wird.

\section{Methodik \\ $\nabla$}

Zur Überprüfung der Prognosevalidität einer derartigen Testbatterie dienen die Daten von $\mathrm{n}=100$ Personen, die am Berufsförderungswerk Eckert in Regenstauf (BFW-E) sowohl die Abklärung der beruflichen Eignung und Arbeitserprobung als auch eine Umschulung durchliefen (vgl. 
Tab. 1 Deskriptive Merkmale der Stichprobe.

\begin{tabular}{|c|c|c|c|c|c|c|}
\hline \multirow{2}{*}{$\begin{array}{l}\text { Merkmal } \\
\text { Alter }^{1}\end{array}$} & \multicolumn{6}{|c|}{ Ausprägung und Wert } \\
\hline & M & SD & Min & Max & fehlend & \\
\hline & $30 ; 2$ & $6 ; 11$ & $18 ; 2$ & $50 ; 0$ & - & \\
\hline \multirow[t]{2}{*}{ Geschlecht } & & weiblich & & männlic & & \\
\hline & & 29 & & 71 & & \\
\hline \multirow[t]{2}{*}{ Ausbildungsrichtung ${ }^{2}$} & BK & HK & IK & ITSE & STF & \\
\hline & 18 & 21 & 20 & 21 & 20 & \\
\hline \multirow[t]{2}{*}{ Familienstand } & ledig & verheiratet & verwitwet & geschieden & fehlend & \\
\hline & 70 & 18 & 2 & 6 & 4 & \\
\hline \multirow[t]{2}{*}{ Kinder } & keine & eins & zwei & drei & vier & fehlend \\
\hline & 72 & 10 & 10 & 3 & 1 & 4 \\
\hline \multirow[t]{2}{*}{ Schulabschluss ${ }^{3}$} & HS & QHS & RS & G & & \\
\hline & 16 & 51 & 30 & 3 & & \\
\hline
\end{tabular}

troniker, STF - Steuerfachangestellte; ${ }^{3}$ HS - Hauptschulabschluss, QHS - Qualifizierter Hauptschulabschluss, RS - Realschulabschluss, G - Abitur

- Tab. 1). Im Einzelnen liegen die Ergebnisse folgender Verfahren vor: Berufsinteressentest II (BIT.II, [1]), Intelligenz-StrukturTest 70 (IST 70, [2]), Figure Reasoning Test (FRT, [3]), Mechanisch-Technischer Verständnistest (MTVT, [4]), Mannheimer Rechtschreibtest (MRT, [5]), Test d2 [6], KonzentrationsLeistungs-Test (KLT, [7]), Test-for-Colour-Deficiency [8], Freiburger Persönlichkeitsinventar (FPI-R, [9]), Berufsbezogener Rechentest (BRT, [10]), die Untertests 3, 4, 8, 9, 10 des Leistungsprüfsystems (LPS, [11]), ein unveröffentlichter Erinnerungstest („Stolpmünde“) und mehrere vom Psychologischen Dienst des BFW-E selbst konzipierte Algebratests. Der Interessenstest BIT.II, der Persönlichkeitstest FPI-R, die Algebratests und der Test-forColour-Deficiency werden im Folgenden für die Prognose nicht weiter berücksichtigt. Interessen und Persönlichkeit haben zwar sicherlich einen Einfluss auf den Umschulungserfolg. Die Tests bestehen allerdings aus einer Vielzahl relativ unabhängiger Skalen, die schwer zusammenfassbar sind und somit die Zahl der Prädiktoren zu groß werden lassen würde. Zudem ist das Ziel der vorliegenden Arbeit, ein auf Leistungsmaßen basierendes Prognosemodell für den Umschulungserfolg zu konstruieren, da metaanalytisch bereits gezeigt werden konnte, dass bivariat bedeutsame Zusammenhänge bestehen (z.B. $[12,13])$. Die Berücksichtigung von Interessen und Persönlichkeit würde außerdem nicht die im BFW-E übliche Diagnostik widerspiegeln. Ebenso gilt dies für andere psychosoziale und sozioökonomische Maße, die zwar bereits eine gewisse prognostische Güte gezeigt haben (z.B. Schulabschluss [14], Alter oder Geschlecht); sie werden auch zugunsten der Vorhersagekraft der psychometrischen Daten ausgeklammert. Die Algebratests sind nicht-standardisierte, unüberprüfte Aufgabensammlungen, über deren Reliabilität nichts ausgesagt werden kann. Eine Farbfehlsichtigkeit wird als unerheblich für den Umschulungserfolg angesehen.

Als Kriterien für den Umschulungserfolg wurden die Noten der Abschlusszeugnisse der Umschulung sowie die Noten der Abschlussprüfung vor der Industrie und Handelskammer (IHK) erhoben. Das eigentliche Ziel einer Umschulungsmaßnahme, die langfristige Reintegration in den Arbeitsmarkt [15], konnte aufgrund der Datengewinnnung der Untersuchung - es wurden bereits erhobene Daten aus den Archiven des BFW-E genutzt - leider nicht überprüft werden. Dies stellt insofern eine Einschränkung der Generalisierung dar, als dass gute Abschlussprüfungen alleine dieses Ziel sicher nicht bedingen. Neben der kognitiven Leistungsfähigkeit und dem schulischen Erfolg haben auch Um-
Tab. 2 Zusammenstellung der einzelnen Testergebnisse nach der Systematik des BFW-E.

\begin{tabular}{|c|c|}
\hline Kategorie & zugehörige Tests $^{1}$ \\
\hline Formallogisches Denken & $\begin{array}{l}\text { LPS 3, LPS 4, FRT gemacht, FRT } \\
\text { richtig* }\end{array}$ \\
\hline Sprachliche Fähigkeiten & IST WA, IST GE, IST SE, IST AN \\
\hline Zahlengebundene Fähigkeiten & $\begin{array}{l}\text { IST ZR A (B*), IST RA, BRT Grund- } \\
\text { rechen, BRT Bruchrechnen, BRT } \\
\text { Prozentrechen, BRT Schlussrech- } \\
\text { nen, Algebratests* (Vortest, nach } \\
\text { Schulung, nach Wochenende) }\end{array}$ \\
\hline Anschauungsgebundenes Denken & $\begin{array}{l}\text { IST WÜ, IST FA, LPS 8, LPS 9, LPS } \\
10\end{array}$ \\
\hline Rechtschreibung & MRT \\
\hline Technisches Verständnis & MTVT \\
\hline Gesamt & IST Gesamtwert \\
\hline Merkfähigkeit & IST ME, Stolpmünde \\
\hline Konzentration & $\begin{array}{l}\text { d2 Gesamt, d2 Fehler } \%, \text { KLT } \\
\text { Gesamt, KLT Fehler } \%\end{array}$ \\
\hline Farbensehen & Ishihara* \\
\hline Berufsinteressen & BIT.II* \\
\hline Persönlichkeit & $\mathrm{FPI}^{*}$ \\
\hline \multicolumn{2}{|c|}{$\begin{array}{l}\text { Anm.: }{ }^{1} \text { LPS } 3 \text { und LPS } 4 \text { - Denkfähigkeit; FRT - allgemeine Intelligenz; IST WA } \\
\text { - Wortauswahl; IST GE - Gemeinsamkeiten; IST SE - Satzergänzung; IST AN - Ana- } \\
\text { logien; IST ZR - Zahlenreihen; IST RA - Rechenaufgaben; BRT - Berufsrechentest; } \\
\text { IST WÜ - Würfelaufgaben; IST FA - Figurenauswahl; LPS 8, LPS } 9 \text { und LPS10 - tech- } \\
\text { nische Begabung; MRT - Rechtschreibung; MTVT - technisches Verständnis; IST ME } \\
\text { - Merkaufgaben; d2 - Aufmerksamkeit; KLT - Konzentration; } \\
{ }^{*} \text { Test wird nicht in die Analyse aufgenommen }\end{array}$} \\
\hline
\end{tabular}

weltfaktoren prädikative Aussagekraft [14]; speziell in dieser Studie waren dies interne Kontrollüberzeugung, Beeinträchtigung durch Schmerz, wahrgenommene soziale Unterstützung und die Art des Umschulungsberufs. Nichtsdestotrotz bereitet ein BFW vorwiegend (zumindest zurzeit noch) auf einen beruflichen Abschluss vor, weshalb der gewählte Fokus als gerechtfertigt erscheint.

Die Prognosevalidität der Testergebnisse wird mittels linearer Regressionsmodelle (z. B. [16-18]) bestimmt. Als abhängige Variable dieser Modelle dienen die Durchschnittsnote des Abschlusszeugnisses sowie der Durchschnitt der drei Noten der IHK-Abschlussprüfung. Es werden mehrere Modelle für unterschiedliche Zusammenstellungen der Testergebnisse als unabhängige Variablen berechnet. 
Tab. 3 Produkt-Momentkorrelationen der abhängigen Variablen IHK-Abschlussnote und der mittleren Umschulungsnote sowie der unabhängigen Variablen Kategorien nach dem BFW-E.

\begin{tabular}{|c|c|c|c|c|c|c|c|c|c|c|c|}
\hline & & (1) & (2) & (3) & (4) & (5) & (6) & (7) & (8) & (9) & (10) \\
\hline (1) & IHK Abschlussnote ${ }^{1}$ & - & $-0,72$ & 0,02 & 0,37 & 0,32 & $-0,01$ & 0,34 & 0,00 & 0,24 & 0,17 \\
\hline (2) & Umschulungsnote & $<0,01$ & - & $-0,13$ & $-0,35$ & $-0,29$ & 0,00 & $-0,39$ & 0,03 & $-0,33$ & $-0,18$ \\
\hline (3) & Formallogisches Denken & 0,42 & 0,10 & - & 0,46 & 0,38 & 0,65 & 0,15 & 0,40 & 0,34 & 0,56 \\
\hline (4) & Sprachliche Fähigkeiten & $<0,01$ & $<0,01$ & $<0,01$ & - & 0,48 & 0,35 & 0,46 & 0,26 & 0,35 & 0,35 \\
\hline (5) & Zahlengebundene Fähigkeiten & $<0,01$ & $<0,01$ & $<0,01$ & $<0,01$ & - & 0,27 & 0,34 & 0,33 & 0,17 & 0,53 \\
\hline (6) & $\begin{array}{l}\text { Anschauungsgebundene } \\
\text { Fähigkeiten }\end{array}$ & 0,48 & 0,49 & $<0,01$ & $<0,01$ & $<0,01$ & - & 0,12 & 0,43 & 0,17 & 0,41 \\
\hline (7) & Rechtschreibung & $<0,01$ & $<0,01$ & 0,06 & $<0,01$ & $<0,01$ & 0,11 & - & 0,07 & 0,46 & 0,29 \\
\hline (8) & Technisches Verständnis & 0,50 & 0,40 & $<0,01$ & $<0,01$ & $<0,01$ & $<0,01$ & 0,24 & - & 0,11 & 0,22 \\
\hline (9) & Merkfähigkeit & 0,02 & $<0,01$ & $<0,01$ & $<0,01$ & 0,05 & 0,04 & $<0,01$ & 0,14 & - & 0,32 \\
\hline (10) & Konzentration & 0,06 & 0,04 & $<0,01$ & $<0,01$ & $<0,01$ & $<0,01$ & $<0,01$ & 0,01 & $<0,01$ & - \\
\hline
\end{tabular}

Anm.: Über der Diagonalen stehen die Produkt-Moment-Korrelationskoeffizienten; unter der Diagonalen das Signifikanzniveau gegen $\mathrm{H}_{0}$ : $\rho=0$. Die den Kategorien zugrunde liegenden Tests sind in Tab. 2 aufgeführt

${ }^{1}$ Für (1) gilt $n=80$, für (2) bis (10) gilt $n=100$; für $n=80$ gilt $p<0,01 \Leftrightarrow|r|>0,29$; für $n=100$ gilt $p<0,01 \Leftrightarrow|r|>0,26$

Die Entscheidungen des Psychologischen Dienstes des BFW-E basieren auf dessen Zusammenfassung der Testergebnisse in unterschiedliche Kategorien (siehe $\bullet$ Tab. 2). In einer umfassenden Darstellung aller Ergebnisse der zugrunde liegenden Studie [19] wurde die Modellpassung dieser Zusammenfassung mittels der Software LISREL 8.5 überprüft. Die Fit-Indizes legen zwar eine nur bedingte Modellpassung nahe (GFI=0,78; RMR $=0,086$, [20]); im Sinne der ökologischen Validität soll aber dennoch die Vorhersagegüte dieser Zusammenfassung überprüft werden. Der Bewertung dieser Kategorien liegt im BFW-E eine fünfstufige Skala zugrunde (unterdurchschnittlich, schlechter Durchschnitt, Durchschnitt, guter Durchschnitt, überdurchschnittlich). Die Einschätzung des Skalenwerts anhand der Einzeltestergebnisse erfolgt sozusagen per Augenmaß. Da ein solches ordinales Maß nur bedingt zur Schätzung von Regressionsmodellen geeignet ist und die einzelnen Test- bzw. Untertestergebnisse zudem als Rohwerte vorliegen, werden zur Bestimmung der Kategoriewerte die z-standardisierten Testwerte benutzt [16]. Der Kategoriewert wird über das arithmetische Mittel der entsprechenden Testwerte berechnet.

Die Regressionsmodelle der Kategorien werden mittels der Backward-Methode des Statistikprogramms SPSS 15 aufgestellt. Dabei wird das Modell gesucht, das mit den wenigsten Variablen den größten Erkenntnisgewinn liefert. Das Kriterium zum Ausschluss einer Variablen ist ihre Bedeutsamkeit für das jeweils überprüfte Modell. Da durch eine höhere Anzahl der Variablen die statistische Unsicherheit der Schätzung steigt, kann ein Modell mit weniger Variablen trotz geringerer Varianzklärung mehr Information liefern [18]. Daher wird das korrigierte Bestimmtheitsmaß $\mathrm{R}^{2}{ }_{\text {korr }}$, das den Anteil der durch die im Modell befindlichen Variablen erklärten Varianz unter Berücksichtigung der Parameteranzahl (J) und der Beobachtungen ( $\mathrm{n}$ ) angibt [16-18], und zwar: $\mathrm{R}^{2}$ korr $=\mathrm{R}^{2}-\mathrm{J}\left(1-\mathrm{R}^{2}\right) /(\mathrm{n}-\mathrm{J}-1)$, als Kriterium herangezogen.

In einem zweiten Schritt wird versucht, ein möglichst aussagekräftiges Modell zur Vorhersage des Umschulungserfolgs mithilfe der Einzeltestergebnisse zu konstruieren. Aufgrund der Neukonzeption des IST [21] werden die Ergebnisse der Subtests und nicht der Gesamtscore in die Berechnungen einbezogen, da hierdurch wenigstens bedingt die Möglichkeit besteht, Parallelen zu ziehen und vergleichbare Ergebnisse zu identifizieren. Da die Backward-Methode für einen Anfangswert von 26 Variablen zu einer Vielzahl sich nur sehr geringfügig unterscheidenden Modelle führen würde, wird für die Suche nach einem geeigneten
Prognosemodell die Stepwise-Funktion von SPSS angewandt [22]. Hierbei wird nach der Variable gesucht, die zu einer größtmöglichen Erhöhung der Varianzklärung führt. Um eine nicht zu geringe Parameterzahl zu erhalten, wird das Signifikanzniveau zur Prüfung der Bedeutsamkeit des Unterschieds in $\mathrm{R}^{2}$ auf $10 \%$ gesetzt. Für einen Vergleich der Güte des resultierenden Modells wird zudem das Modell mit allen Testergebnissen als Prädiktoren angegeben.

\section{Ergebnisse \\ $\nabla$}

\section{Das Modell des BFW Eckert}

Die Umschulungsnoten (Schulnoten zwischen 1 und 6) haben einen Mittelwert von 2,34 (SD =0,69). Die gemittelten Noten der IHK-Prüfung (3 Noten, bestens 100 Punkte) liegen im Schnitt bei $75,1(S D=8,7 ; n=80)$. Die beiden abhängigen Variablen korrelieren mit $-0,72$. Die Kollinearitätsstatistik (vgl. [19]) weist zwar teilweise hohe, insgesamt jedoch keine kritischen Korrelationen der Kategorien untereinander aus (z. B. Formallogisches Denken mit Anschauungsgebunden Fähigkeiten 0,65, mit Konzentration 0,56 und mit Sprachlichen Fähigkeiten 0,46; vgl. @ Tab. 3). Das Regressionsmodell mit allen Kategorien (Modell 1) erklärt 19\% (unkorrigiert 25\%) der Varianz der Umschulungsnoten und 17\% (unkorrigiert 25\%) der Varianz der IHK-Abschlussnoten (vgl. - Tab. 4 und 5). Die den Modellen zugehörigen Bestimmtheitsmaße werden in $\bullet$ Tab. 6 angegeben. Sowohl der schrittweise Vergleich der Modelle zur Vorhersage der Umschulungsnoten als auch der Vergleich von Modell 1 und Modell 7 zeigen keine bedeutsamen Unterschiede bezüglich der Varianzaufklärung $\left(\Delta \mathrm{R}^{2}{ }_{1-7}=0,063, \mathrm{~F}[6,91]=1,27, \mathrm{p}=0,28\right)$. Das korrigierte Bestimmtheitsmaß ist für die Modelle 3 und 4 am größten. Für die Vorhersage der IHK-Abschlussnoten gilt dies in gleicher Weise, außer dass bereits in Modell 6 alle Variablen statistisch bedeutsame Beta-Gewichte aufweisen. Die Modelle 1-6 unterscheiden sich nicht in ihrer Varianzklärung sowohl beim schrittweisen Vergleich als auch beim Vergleich der Modelle 1 und $6\left(\Delta \mathrm{R}^{2}{ }_{1-6}\right.$ $=0,039, F[5,71]=0,74, p=0,60)$. Das höchste korrigierte $\mathrm{Be}$ stimmtheitsmaß liegt für die Modelle 4 und 5 vor.

\section{Das Modell mittels Einzelvariablen}

Die Regressionsgleichungen zur Schätzung der Umschulungsnoten bzw. der IHK-Abschlussnoten werden in $\bullet$ Tab. 7 dargestellt. Das Modell zu den Umschulungsnoten erklärt 14\% der Varianz 

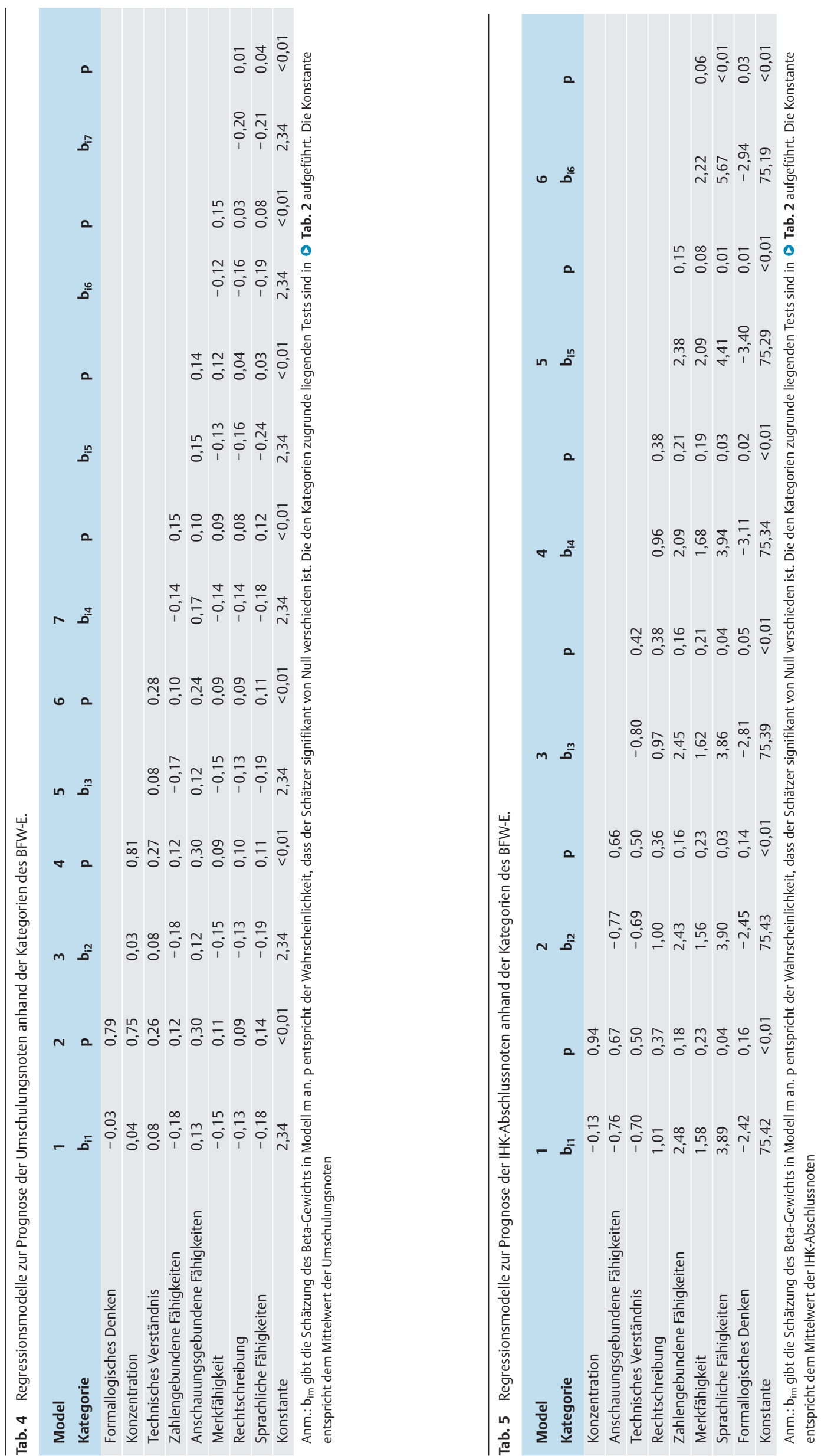
Tab. 6 Modellzusammenfassungen für die Schätzung der Umschulungsnoten (links) und die Schätzung der IHK-Abschlussnoten (rechts) anhand der Kategorien des BFW-E.

\begin{tabular}{|c|c|c|c|c|c|c|c|}
\hline \multirow[b]{2}{*}{ Modell } & \multicolumn{3}{|c|}{ Umschulungsnote } & \multicolumn{4}{|c|}{ IHK-Abschlussnote } \\
\hline & $\mathbf{R}$ & $\mathbf{R}^{2}$ & $\mathbf{R}_{\text {korr }}^{2}$ & Modell & $\mathbf{R}$ & $\mathbf{R}^{2}$ & $\mathbf{R}_{\text {korr }}^{2}$ \\
\hline 1 & 0,502 & 0,252 & 0,186 & 1 & 0,500 & 0,250 & 0,166 \\
\hline 2 & 0,501 & 0,251 & 0,195 & 2 & 0,500 & 0,250 & 0,177 \\
\hline 3 & 0,501 & 0,251 & 0,203 & 3 & 0,498 & 0,248 & 0,186 \\
\hline 4 & 0,491 & 0,241 & 0,201 & 4 & 0,491 & 0,241 & 0,190 \\
\hline 5 & 0,474 & 0,225 & 0,192 & 5 & 0,483 & 0,233 & 0,192 \\
\hline 6 & 0,455 & 0,207 & 0,182 & 6 & 0,460 & 0,211 & 0,180 \\
\hline 7 & 0,435 & 0,189 & 0,173 & & & & \\
\hline
\end{tabular}

Anm.: R - multiple Korrelation, $\mathrm{R}^{2}$ - Bestimmtheitsmaß, $\mathrm{R}^{2}$ korr - korrigiertes Bestimmtheitsmaß; kein Modell weist einen statistisch signifikanten Unterschied bezüglich der Varianzklärung auf

(unkorrigiert 36\%, F[26,73]=1,61, p=0,059). Das Modell zu den IHK-Abschlussnoten erklärt 15\% der Varianz (unkorrigiert $43 \%$, $\mathrm{F}[26,53]=1,52, \mathrm{p}=0,099)$. In ○ Tab. 8 werden die Veränderungen der Varianzklärung durch Hinzunahme der einzelnen jeweils besten Variablen (vgl. @ Tab. 9) dargestellt. Es zeigt sich, dass nach Aufnahme von fünf Variablen (Umschulungsnoten) bzw. vier Variablen (IHK-Abschlussnoten) keine weitere Variable gefunden werden kann, die zu einer bedeutsamen Steigerung der Varianzklärung beitragen kann. Die resultierenden Modelle klären 25\% (Umschulungsnoten) bzw. 27\% (IHK-Abschlussnoten) der Varianz (unkorrigiert 29\% bzw. 31\%). Der Vergleich dieser Modelle mit den Modellen aller Testergebnisse zeigt, dass die Verringerung der unkorrigierten Varianz statistisch nicht signifikant ist (Umschulungsnoten: $\Delta R^{2}=7 \%, F[21,73]=0,40, p=0,989$; IHK-Abschlussnoten: $\left.\Delta R^{2}=12 \%, F[22,53]=0,50, p=0,960\right)$. Durch die Reduzierung der Prädiktoren werden die jeweiligen Modelle bzw. die Schätzung der Varianzklärung erst bedeutsam (vgl. - Tab. 7 und 9).

\section{Diskussion \\ $\nabla$}

Die Reduktion der Prädiktoren zur Vorhersage des Umschulungserfolgs mittels der Kategorien des BFW-E ist bei Betrachtung der bivariaten Korrelationen der Kategorien in $\bullet$ Tab. 2 nachvollziehbar. Zum einen bestehen - bis auf eine Ausnahme - keine bedeutsamen Korrelationen zwischen den weggefallenen Prädiktoren und den Kriteriumsvariablen. Zum anderen lassen die teilweise hohen Korrelationen der Prädiktoren untereinander - trotz tolerierbarer Kollinearität ( $\bullet$ Tab. 3, vgl. [19]) - ein gewisses Maß an Redundanz erahnen. Auffällig sind vor allem die unerwarteten Werte der Kategorie Formallogisches Denken. Bivariat besteht kein Zusammenhang zwischen diesem Maß für sprachfreie Intelligenz und dem Umschulungserfolg $\left(r_{\mathrm{FD}-\mathrm{IHK}}=0,02 ; \mathrm{r}_{\mathrm{FD}-\mathrm{US}}=-0,13\right)$. Im Regressionsmodell zur IHK-Abschlussnote nimmt diese Kategorie allerdings eine äußerst prominente Rolle ein: Aufgrund der Korrelationen zwischen Formallogischem Denken und Sprachlichen Fähigkeiten sowie Merkfähigkeit ist zu erwarten, dass Personen mit hoher allgemeiner Intelligenz auch in den beiden anderen Bereichen leistungsfähiger bzw. Personen mit niedriger allgemeiner Intelligenz weniger leistungsfähig sind. Da sich dieser Zusammenhang nicht in gleicher Weise in den Abschlussnoten widerspiegelt, wird eine Korrektur nötig, um die Linearität des Modells darstellen zu können, die sich in dem statistisch negativen Einfluss des Formallogischen Denkens auf die Prognose darstellt $(b=-2,94$, - Tab. 5). Bei der Interpretation der Werte ist zu beachten, dass
Tab. 7 Regressionsmodelle zur Prognose der Umschulungsnoten (links) und der IHK-Abschlussnoten (rechts) mittels aller z-standardisierter Einzeltestergebnisse.

\begin{tabular}{|c|c|c|c|c|}
\hline & \multicolumn{2}{|c|}{ Umschulungs-note } & \multicolumn{2}{|c|}{ IHK-Abschlussnote } \\
\hline & $b_{i, \text { us }}$ & $\mathbf{p}$ & 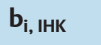 & $\mathbf{p}$ \\
\hline Konstante & 2,344 & $<0,001$ & 75,684 & $<0,001$ \\
\hline LPS3 & $-0,159$ & 0,113 & $-1,245$ & 0,405 \\
\hline LPS4 & 0,089 & 0,396 & $-0,035$ & 0,981 \\
\hline FRT & 0,012 & 0,923 & $-0,703$ & 0,679 \\
\hline MRT & $-0,183$ & 0,045 & 1,124 & 0,385 \\
\hline IST SE & $-0,037$ & 0,653 & 2,879 & 0,018 \\
\hline IST AN & $-0,056$ & 0,550 & 0,651 & 0,635 \\
\hline IST WA & $-0,051$ & 0,541 & $-1,414$ & 0,257 \\
\hline IST GE & $-0,007$ & 0,932 & 0,998 & 0,434 \\
\hline IST ZR & $-0,027$ & 0,814 & $-0,103$ & 0,949 \\
\hline IST RA & 0,002 & 0,988 & 0,224 & 0,906 \\
\hline BRT G & 0,009 & 0,926 & 1,032 & 0,465 \\
\hline BRT B & $-0,144$ & 0,146 & 1,899 & 0,193 \\
\hline BRT P & $-0,119$ & 0,262 & 1,641 & 0,342 \\
\hline BRT S & 0,040 & 0,707 & $-2,086$ & 0,172 \\
\hline MTVT & 0,041 & 0,648 & $-0,030$ & 0,982 \\
\hline LPS10 & 0,024 & 0,795 & $-0,091$ & 0,947 \\
\hline IST FA & 0,012 & 0,890 & 0,729 & 0,560 \\
\hline IST WÜ & 0,094 & 0,271 & 0,864 & 0,497 \\
\hline LPS8 & 0,035 & 0,739 & $-2,279$ & 0,156 \\
\hline LPS9 & $-0,037$ & 0,665 & 0,331 & 0,782 \\
\hline IST ME & $-0,136$ & 0,108 & 2,514 & 0,047 \\
\hline STOLMÜNDE & $-0,047$ & 0,620 & $-0,747$ & 0,583 \\
\hline D2 GESAMT & $-0,085$ & 0,308 & 0,579 & 0,625 \\
\hline D2\% RICHTIG & $-0,008$ & 0,922 & 1,062 & 0,352 \\
\hline KLT GESAMT & 0,155 & 0,163 & $-0,200$ & 0,901 \\
\hline KLT\% RICHTIG & 0,036 & 0,684 & $-1,162$ & 0,342 \\
\hline $\mathrm{R}_{\text {korr }}^{2}$ & 0,138 & 0,059 & 0,146 & 0,099 \\
\hline
\end{tabular}

Anm.: $b_{i}$, gibt die Schätzung des Beta-Gewichts für das Modell zur Prognose der Umschulungsnote (US) bzw. IHK-Abschlussnote (IHK) an. p entspricht der Wahrscheinlichkeit, dass der Schätzer signifikant von Null verschieden ist. Die Konstante entspricht dem Mittelwert des Kriteriums

die Variablen standardisiert sind, d.h. ihr Mittelwert ist 0, ihre Standardabweichung 1. Ausgangspunkt im Regressionsmodell ist der Mittelwert (also die Konstante, in Modell 6 gilt beispielsweise $b_{0}=75,19$ ). Für eine Person, die in allen drei Kategorien durchschnittlich ist, würde also genau dieser Wert erwartet. Da sich bei Personen, deren Sprachliche Fähigkeiten deutlich besser als der Durchschnitt sind, dieser Vorsprung nicht eins-zu-eins in der IHK-Note widerspiegelt, wird diese Diskrepanz durch das negative Beta-Gewicht des Formallogischen Denkens aufgehoben. Das Weglassen der Variable würde einen Verlust von 5\% 
Tab. 8 Modellzusammenfassungen für die Schätzung der Umschulungsnoten (oben) und die Schätzung der IHK-Abschlussnoten (unten) anhand der durch die Stepwise-Methode ausgewählten Einzeltestergebnisse.

\begin{tabular}{|c|c|c|c|c|c|c|c|}
\hline \multicolumn{8}{|c|}{ Umschulungsnote } \\
\hline Modell & $\mathbf{R}$ & $\mathrm{R}^{2}$ & $\mathbf{R}_{\text {korr }}^{2}$ & $\Delta R^{2}$ & $\mathbf{F}$ & df & $\mathbf{p}$ \\
\hline 1 & 0,393 & 0,154 & 0,146 & 0,154 & 17,90 & $(1,98)$ & $<0,001$ \\
\hline 2 & 0,456 & 0,208 & 0,192 & 0,054 & 6,56 & $(1,97)$ & 0,012 \\
\hline 3 & 0,490 & 0,240 & 0,217 & 0,032 & 4,10 & $(1,96)$ & 0,046 \\
\hline 4 & 0,519 & 0,269 & 0,238 & 0,028 & 3,70 & $(1,95)$ & 0,057 \\
\hline 5 & 0,539 & 0,291 & 0,253 & 0,022 & 2,87 & $(1,94)$ & 0,093 \\
\hline \multicolumn{8}{|c|}{ IHK-Abschlussnote } \\
\hline Modell & $\mathbf{R}$ & $R^{2}$ & $\mathbf{R}_{\text {korr }}^{2}$ & $\Delta R^{2}$ & $\mathbf{F}$ & df & $\mathbf{p}$ \\
\hline 1 & 0,439 & 0,193 & 0,183 & 0,193 & 18,67 & $(1,78)$ & $<0,001$ \\
\hline 2 & 0,493 & 0,243 & 0,224 & 0,050 & 5,11 & $(1,77)$ & 0,027 \\
\hline 3 & 0,528 & 0,279 & 0,250 & 0,035 & 3,71 & $(1,76)$ & 0,058 \\
\hline 4 & 0,554 & 0,307 & 0,270 & 0,029 & 3,09 & $(1,75)$ & 0,083 \\
\hline
\end{tabular}

Varianzklärung bedeuten und wird daher unterlassen. Zur Vorhersage des Umschulungserfolgs einer kaufmännischen Ausbildung ist die Berücksichtigung der nonverbalen Intelligenz als Mediator (mit der Konsequenz eines Suppressionseffektes [16]) also nötig. Der Schluss, dass ein niedriger Wert gleichzeitig ein besseres Umschulungsergebnis bedingt, darf allerdings nicht gezogen werden.

Insgesamt wird deutlich, dass zur Vorhersage des Umschulungserfolgs einer kaufmännischen Ausbildung folgende Merkmale (im Interpretationssetting des BFW-E) relevant und aussagekräftig sind (vgl. @ Tab. 4 und 5): Sprachliche Fähigkeiten, Merkfähigkeit, Rechtschreibung und in einem gewissen Maße Zahlengebundene Fähigkeiten. Konzentration, Technisches Verständnis und Anschauungsgebundene Fähigkeiten haben - thematisch bedingt - erwartungsgemäß keinen bzw. keinen direkten Einfluss. Unter Berücksichtigung von vier Kategorien können jeweils 19\% der Varianz der Erfolgskriterien erklärt werden ( Tab. 6). Dieses Ergebnis bestätigt die bisher gefundenen Resultate, nach denen die unterschiedlichsten Maße allgemeiner und spezieller Intelligenz, allerdings vorwiegend in bivariaten Vergleichen, in diesen Bereichen korrelieren (z.B. [12]: zwischen 0,27 und 0,61 ; [23]: zwischen 0,40 und 0,60 , speziell auf den Ausbildungserfolg bezogen [13]: zwischen 0,37 und 0,54; für eine Übersicht vgl. [24]: Zusammenstellung vieler Metaanalysen, Korrelationen zwischen 0,42 und 0,51; durch Quadrieren der Korrelationskoeffizienten erhält man das Bestimmtheitsmaß).

Aufgrund der hohen Parameteranzahl bei der gleichzeitigen Berücksichtigung aller Testergebnisse zur Prognose des Umschulungserfolgs sind die Schätzungen der erklärbaren Varianz nicht einmal statistisch bedeutsam (vgl. 0 Tab. 7). Durch die Beachtung einiger ausgewählter Variablen ist eine statistisch bessere Vorhersage des Umschulungserfolgs möglich als durch das Konglomerat der Tests in Kategorien: Fünf Variablen (Umschulungsnoten) bzw. vier Variablen (IHK-Abschlussnoten) klären 25\% bzw. 27\% der Varianz (vgl. @ Tab. 8). Diese Werte entsprechen dem empirischen Maximum der erwähnten Studien. Auch bei der Modellierung des Umschulungserfolgs mittels einzelner Testergebnisse erweist es sich offenbar als statistisch notwendig, die höhere allgemeine intellektuelle Leistungsfähigkeit durch einen entgegen der Erwartung gerichteten Prädiktor zu korrigieren. Im Modell zur Vorhersage der Umschulungsnoten ist dies der IST-Subtest Würfelaufgaben, bei den IHK-Abschlussnoten der
FRT (vgl. Tab. 9). Diese beiden Variablen korrelieren mit 0,37. Auch inhaltlich spiegeln sich die Ergebnisse der Kategorie-Modelle wider: Zur Vorhersage des Umschulungserfolgs ist die Berücksichtigung einer sprachlichen Komponente (MRT bzw. IST SE), der Merkfähigkeit (IST ME) und des mathematischen Basiswissens (BRT B) nötig. Offenbar ist Bruchrechnen also ein guter Indikator für schulische Mathematikfähigkeit.

Durch den Versuch, das jeweils andere Kriterium durch das Modell des anderen zu schätzen (vgl. @ Tab. 10), wird deutlich, dass die beiden Umschulungserfolgsmaße zwar zusammenhängen $(\mathrm{r}=-0,72)$ und inhaltlich durch ähnliche Merkmale bedingt sind, für gute Schulnoten aber die Rechtschreibung und für einzelne Prüfungsnoten das Sprachverständnis die größere Rolle spielen. Auffällig ist auch, dass die Schätzung der nonverbalen Intelligenz durch den FRT keinen prognostischen Wert bezüglich der Umschulungsnote besitzt.

Insgesamt wird deutlich, dass zur Vorhersage des Umschulungserfolgs einer kaufmännischen Ausbildung nur wenige Variablen nötig sind. Vor allem sprachliche Fähigkeiten und mathematisches Basiswissen sind geeignete Prädiktoren für gute Umschulungsergebnisse (vgl. auch [25]). Im Bereich der Erwachsenenbildung könnten an dieser Stelle noch die Ergebnisse der Rolle des Vorwissens zur Vorhersage des Schulerfolgs diskutiert werden; schließlich hat sich im schulischen Umfeld Vorwissen mit zunehmendem Alter der Probanden als immer besserer Prädiktor der Schulleistung im Vergleich zur Intelligenz erwiesen [26].

Zuletzt sei noch darauf hingewiesen, dass die Prognose des Umschulungserfolgs nur ein Aspekt der Nutzung der Daten der Abklärung der beruflichen Eignung und Arbeitserprobung ist. Sie dienen ferner der Einschätzung der Eignung für verschiedene berufliche Ausbildungsrichtungen, was den Einsatz einer umfassenden Testbatterie rechtfertigt. Außerdem sei hervorgehoben, dass kognitive Kompetenzen und Vorwissen nur einen Faktor für Umschulungserfolg darstellen. Mindestens ebenso wichtig sind motivationale, familiäre und situative Faktoren (sog. konative Determinanten) sowie das Selbstbild [27], die in vorliegender Arbeit nicht berücksichtigt wurden. 


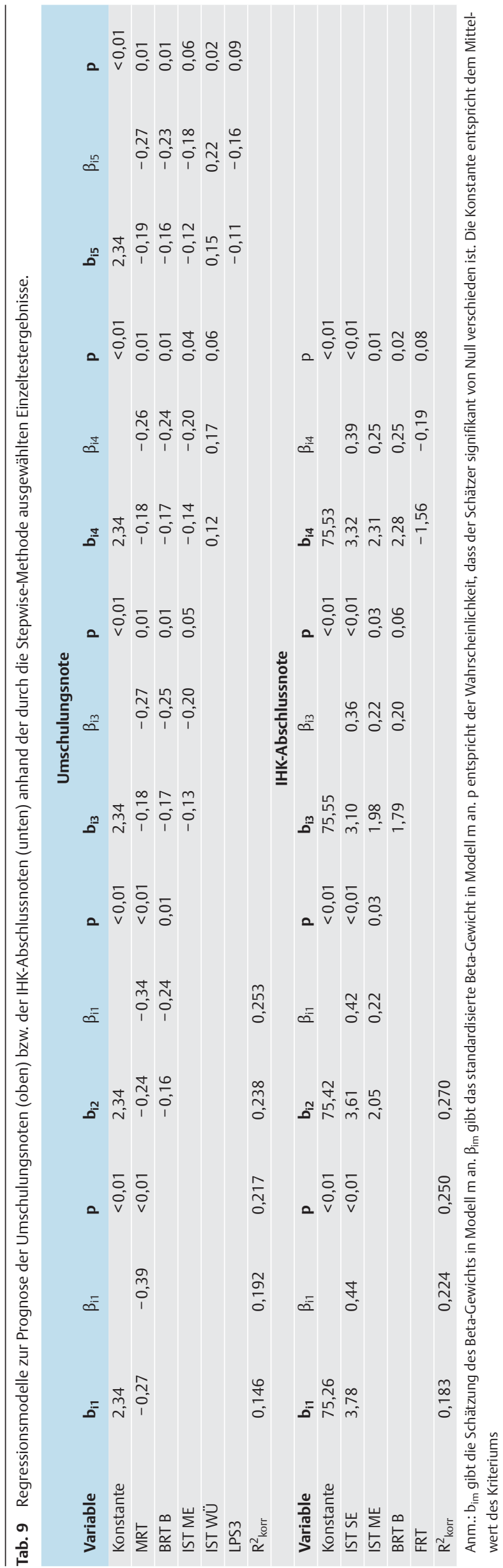

Tab. 10 Regressionsmodelle zur Prognose der Umschulungsnoten (links) bzw. der IHK-Abschlussnoten (rechts) anhand des jeweils durch das andere geschätzte Modell der Einzeltestergebnisse.

\begin{tabular}{|llllll}
\multicolumn{3}{c}{ Umschulungsnote } & \multicolumn{3}{c}{ IHK-Abschlussnote } \\
& & & & & \\
& $\mathbf{b}_{\mathbf{i}, \text { us }}$ & $\mathbf{P}$ & & $\mathbf{b}_{\mathbf{i}, \text { IHK }}$ & $\mathbf{p}$ \\
\hline Konstante & 2,34 & $<0,01$ & Konstante & 75,56 & $<0,01$ \\
\hline IST SE & $-0,10$ & 0,13 & IST ME & 1,61 & 0,11 \\
\hline IST ME & $-0,20$ & 0,00 & BRT B & 2,40 & 0,02 \\
\hline BRT B & $-0,19$ & 0,01 & MRT & 1,84 & 0,07 \\
\hline FRT & 0,02 & 0,80 & IST WÜ & $-0,25$ & 0,79 \\
\hline & & & LPS3 & $-0,72$ & 0,48 \\
\hline$R^{2}$ korr & 0,170 & $<0,01$ & R $^{2}$ korr & 0,156 & $<0,01$ \\
\hline
\end{tabular}

Anm.: $b_{i}$. gibt die Schätzung des Beta-Gewichts für das Modell zur Prognose der Umschulungsnote (US) bzw. IHK-Abschlussnote (IHK) an. p entspricht der Wahrscheinlichkeit, dass der Schätzer signifikant von Null verschieden ist. Die Konstante entspricht dem Mittelwert des Kriteriums

\section{Kernbotschaft}

Der Umschulungserfolg einer kaufmännischen Ausbildung hängt vorwiegend von der sprachlichen Kompetenz und vom Vorhandensein schulischen Vorwissens ab. Nonverbale Intelligenz steht hingegen in keinem direkten korrelativen Zusammenhang zu den Noten einer Umschulung.

\section{Literatur}

1 Irle M, Allehoff $W$. Berufsinteressentest II (BIT II). Göttingen: Hogrefe; 1984

2 Amthauer R. IST 70. Göttingen: Hogrefe; 1970

3 Daniels JC. F-R-T. Figure Reasoning Test. Göttingen: Hogrefe; 1962

4 Lienert GA. Mechanisch-Technischer Verständnistest. Göttingen: Hogrefe; 1958

5 Jäger $R$, Jundt E. Mannheimer Rechtschreib-Test (M-R-T). Göttingen: Hogrefe; 1981

6 Brickenkamp R. Test d2. Göttingen: Hogrefe; 1994

7 Düker H, Lienert GA. Konzentrations-Leistungs-Test. Göttingen: Hogrefe; 1959

8 Ishihara S. The Series of Plates designed as a Test for Colour-Deficiency. Tokyo: Kanehara; 1997

9 Fahrenberg J, Hampel R, Selg H. FPI-R: Das Freiburger Persönlichkeitsinventar. Göttingen: Hogrefe; 2001

10 Balser H, Ringsdorfer O, Traxler A. Berufsbezogener Rechentest. Göttingen: Hogrefe; 1986

11 Horn W. Leistungsprüfsystem. Göttingen: Hogrefe|; 1962

12 Hunter JE, Hunter RF. Validity and utility of alternative predictors of job performance. Psychological Bulletin 1984; 96 (1): 72-98

13 Hülsheger UR, Maier GW, Stumpp T, Muck PM. Vergleich kriteriumsbezogener Validitäten verschiedener Intelligenztests zur Vorhersage von Ausbildungserfolg in Deutschland: Ergebnisse einer Metaanalyse. Zeitschrift für Personalpsychologie 2006; 5: 145-162

14 Köster T, Fehr M, Slesina W. Zur Eingliederung von Rehabilitanden in das Erwerbsleben nach Umschulung in Berufsförderungswerken - ein Prognosemodell. Rehabilitation 2007; 46 (5): 258-265

15 Schmidt C, Froböse I, Schian H-M. Berufliche Rehabilitation in Bewegung - Herausforderungen und Perspektiven. Rehabilitation 2006; 45 (4): 194-202

16 Bortz J. Statistik für Sozialwissenschaftler. Berlin: Springer; 2005

17 Backhaus K, Erichson B, Plinke W, Weiber R. Multivariate Analysemethoden. Berlin: Springer; 2003

18 Fahrmeir L, Hamerle A, Tutz G. Multivariate statistische Verfahren. Berlin: de Gruyter; 1996

19 Kreuzpointner L. Statistische Analysen eignungsdiagnostischer Daten: Anwendung multivariater Verfahren im Rahmen einer Untersuchung der Eignungsdiagnostik des Berufsförderungswerks Eckert. Saarbrücken: VDM Verlag Dr. Müller; 2008

20 Bollen KA. Structural Equations with latent Variables. New York: Wiley; 1989 
21 Amthauer R, Brocke B, Liepmann D et al. IST 2000 R. Göttingen: Hogrefe; 2001

22 Diehl JM, Staufenbiel T. Statistik mit SPSS für Windows: Version 15. Eschborn bei Frankfurt am Main: Klotz; 2001

23 Funke J. Computergestützte Arbeitsproben: Begriffsklärung, Beispiele sowie Entwicklungspotentiale. Zeitschrift für Arbeits- und Organisationspsychologie 1993; 37: 119-129

24 Fraser BJ, Walberg HJ, Welch WW, Hattie JA. Syntheses of educational productivity research. International Journal of Educational Research 1993; 11: 145-252
25 Schmidt JU. Zur Validität von Schulkenntnistests. Zeitschrift für Arbeits- und Organisationspsychologie 1993; 38: 112-117

26 Blum BS. Human Characteristics and School Learning. New York: Mc Graw Hill; 1993

27 Helmke A, Weinert FE. Bedingungsfaktoren schulischer Leistungen. In: Weinert FE, Hrsg. Psychologie des Unterrichts und der Schule. Göttingen: Hogrefe; 1993; 71-176 